\title{
Fabrication and Magnetic Properties of Co Nanostructures in AAO Membranes
}

\author{
J.-S. Jung $,{ }^{\ddagger}, \circ$ L. Malkinski, ${ }^{\ddagger}$ J.-H. Lim, ${ }^{\ddagger}$ M. Yu, ${ }^{\ddagger}$ C. J. O'Connor,${ }^{\ddagger}$ H.-O. Lee, ${ }^{\dagger}$ and E.-M Kim ${ }^{\dagger .}$ \\ ${ }^{\dagger}$ Department of Chemistry, Kanghung National Chwersity, Kangnung 210-702. Korea. E-mail: jjscmigkanghung.ac.kr \\ Adwanced Materials Research Institute, Unversity of New Orleans, New' Orleans. LA 701+8, USA \\ $\$$ Korea Basic Science Institute, Gangneung 210-720, Korea \\ Received December 20,2007
}

\begin{abstract}
Nanoporous AAO (Anodic Aluminum Oxide) membranes have many advantages as a template for variety of magnetic materials. Materials can be embedded into the pores by electrodeposition, sputtering or magneticfield-assisted infiltration of magnetic nanoparticles. This work focuses on the fabrication of the magnetic structures in the AAO templates by electrodeposition. Our method allows the controlled growth of Co nanostructures within the porous alumina membrane in the form of dots, rods and long wires. The shape of Co nanostructures has been investigated by field emission scanuing electron microscope (FESEM). The magnetic hysteresis loops of Co nanostructures were measured using SQUID at $5 \mathrm{~K}$ and $300 \mathrm{~K}$. The magnetic properties of the Co nanostructures are proportional to their aspect ratios and can be controlled by changing the aspect ratios.
\end{abstract}

Key Words : Co nanostnuctures. Nanoporous AAO. Electrodeposition

\section{Introduction}

The fabrication of nanostructured materials, such as nanodots and nanowires, is of considerable interest due to their various potential applications in information technology, biotechnology, medicine, or environmental engineering. ${ }^{1 \cdot 4}$ The morphology of various nanostructures can greatly influence their properties. Magnetic materials is one important example where magnetic response is quite sensitive to variability in the sizes, shapes. and spacing of the nanostructured materials. Nanoscaled materials can be usually produced by performing chenical or electrochemical reaction techniques. Among them, electrodeposition of nanodots and nanowires in self-assembled AAO menbrane due to the highly ordered pores, density, and the controllable pore diameter. is a simple. low cost and high throughout technique to fabricate arrays of nanostructures. ${ }^{5}$ The size of the magnetic metal nanostructures depends on deposition conditions such as the current density and the deposition time. The elongated shape of nanorods or nanowires. especially those of cobalt, gives rise to significant magnetostatic effects that impact coercivity and uniaxial shape anisotropy.

\section{Experiment}

AAO template was synthesized by two-step anodization in a $0.2 \mathrm{M} \mathrm{H}_{2} \mathrm{C}_{2} \mathrm{O}_{4}$ solution. ${ }^{6} \mathrm{Co}$ nanostructures were deposited in the pores of AAO by DC electrolysis in an electrolyte of the following conposition: $\mathrm{CoSO}_{4} \cdot 7 \mathrm{H}_{2} \mathrm{O}(240 \mathrm{~g} / \mathrm{L})$. boric acid $(40 \mathrm{~g} / \mathrm{L})$ and ascorbic acid $(1 \mathrm{~g} / \mathrm{L})$. Silver conducting layer for electrodeposition of cobalt was coating onto the one side of through AAO hole. The $\mathrm{pH}$ value of electrolyte was maintained at 3.19 and then the electrolysis was conducted at $25^{\circ} \mathrm{C}$. using DC current of $3.4 \mathrm{~mA}$ and silver counterelectrodes. Cobalt nanostructures were fabricated by simply controlling the deposition time in the range from 5 $\mathrm{sec}$ to $15 \mathrm{~min}$. The resulting lengths of nanodots. nanorods, nanowires was $80 \mathrm{~mm} .270 \mathrm{~nm}, 2.6 / \mathrm{mm}$. relatively.

Field-emission scaming electron microscopy (FESEM) imaging was performed on a Hitachi $\$-4200$ microscope. Powder X-ray diffraction (XRD) data were collected on a Bruker D8 advanced system equipped with copper radiation and a graphite monochromator. Magnetic properties were studied with a Quantum Design MPMS-5S superconducting Quantum Interference Device Magnetometer at $300 \mathrm{~K}$. A general discussion of the magnetic susceptibility measurements and calibration techniques is described in detail elsewhere. ${ }^{7}$ The ferromagnetic resonance (FMR) was measured at room temperature using an X-band Bruker EMX300 electron paramagnetic resonance (EPR) spectrometer.

\section{Results and Discussion}

Morphology of the Co nanostructures is shown in field emission scanning electron microscopy (FESEM) images in Figure 1. Figure I(a), (b) and (c) show FESEM micrographs of $\mathrm{Co}$ nanostructures after the AAO has been removed. The average pore diameter of $\mathrm{AAO}$, which determines the diameter of the wires, was $80 \mathrm{~mm}$. The lengths of electrodeposited nanodots (a), nanorods (b). nanowires (c) are 80 nm. $270 \mathrm{~nm} .2 .6 \mathrm{~mm}$, respectively. Both the diameter and lengths of these Co nanostructures are very uniform. Images presented in Figures l(d). (e) and (f) were observed after splitting the AAO membranes by bending them. These side views exhibit well-ordered $\mathrm{Co}$ nanostructures. Electrochemically grown Co nanowires generally have either fcc or hep structure. ${ }^{8}$ In our work. Co nanowires have mostly hep structure, as evidenced by X-ray diffraction shown in Figure 2.

The hysteresis loops measured at room temperature are 

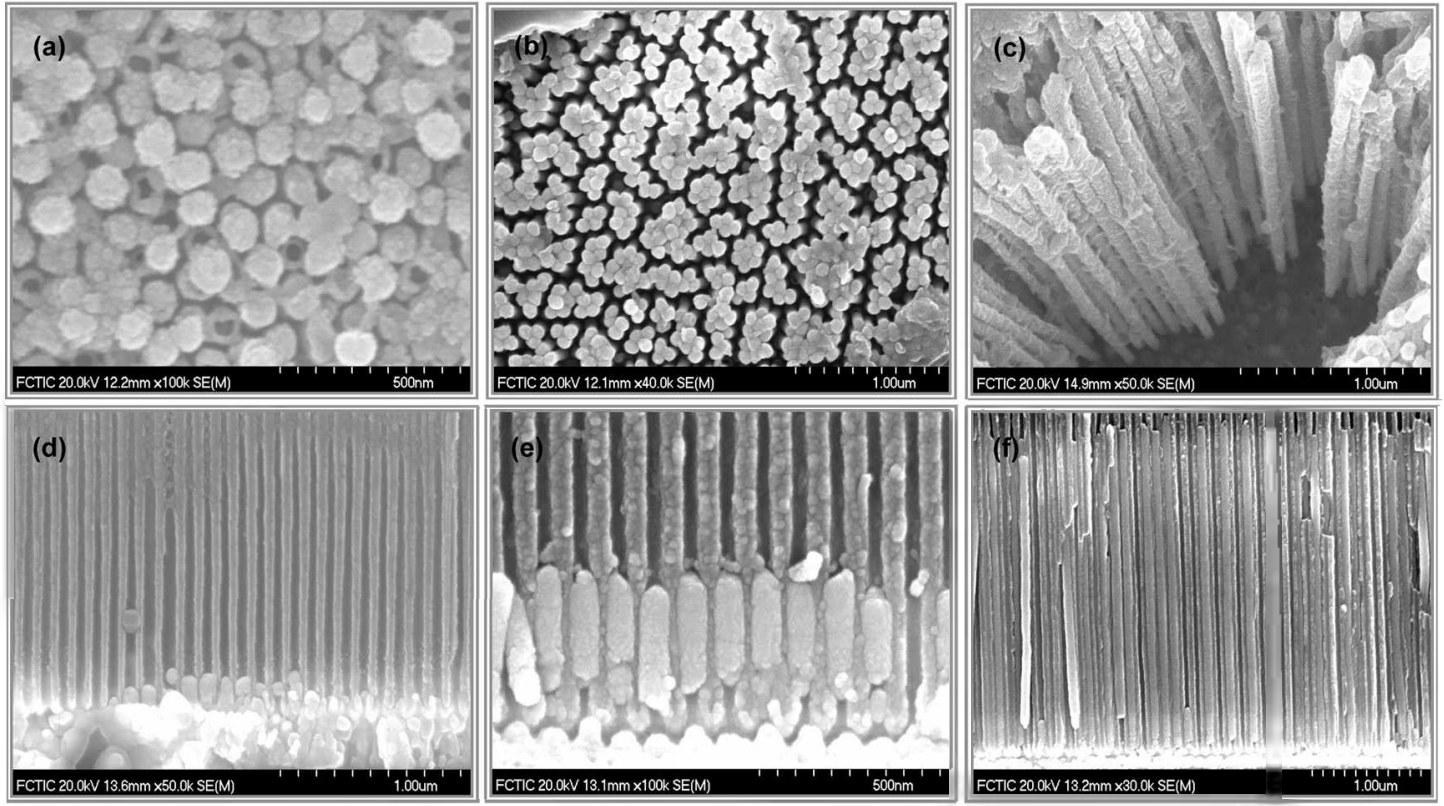

Figure 1. The SEM inages of Co (a) nanodots (b) nanorods (c) nanowires after removal of the AAO and (d) nanodots (e) nanorods ( $f$ ) nannowires cross section in AAO.

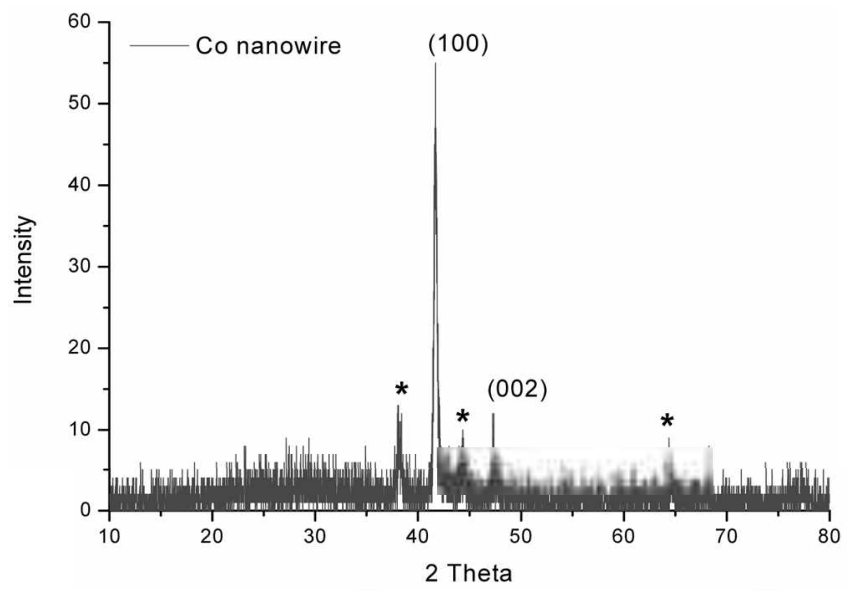

Figure 2. XRD pattens of Co nanowires (* indicate Ag peaks on the AAO pore bottom); the major Co reflections are those of the hep structure.

shown in Figure 3. The magnetization hysteresis loops of the nanodots were almost independent of the direction of the applied magnetic field. However, distinct difference in magnetization process were observed for the elongated structures: rods and wires. Square magnetic hysteresis loops for the direction of the field nomal to the AAO membrane plane, and tilted hysteresis loops with much smaller coercivity in case of the field applied parallel to the membrane plane are characteristic of systems with uniaxial anisotropy. Since the direction of the uniaxial anisotropy coincides with the long axis of the rods and wires, it can be postulated that the shape anisotropy due to elongated shape of the rods has pronounced effect on magnetic properties of these nanostructures. ${ }^{9,11}$ The remanence to saturation ratio $\left(\mathrm{M}_{\mathrm{r}} / \mathrm{M}_{s}\right)$ of nanodot is only 0.16 , and coercivity $40 \mathrm{Oe}$. The remanence ratio. which characterizes the squareness of the hysteresis loops. increases to 0.27 for nanorods and to 0.21 for the wires. whereas the coercivity increases to 480 Oe for nanorods and to 940 Oe for nanowires. which is more than 20 times that of the dots. Unlike the remanence ratio. the coercivity shows monotonic increase with the aspect ratio change of Co nanostructures from nanodots to nanowires.
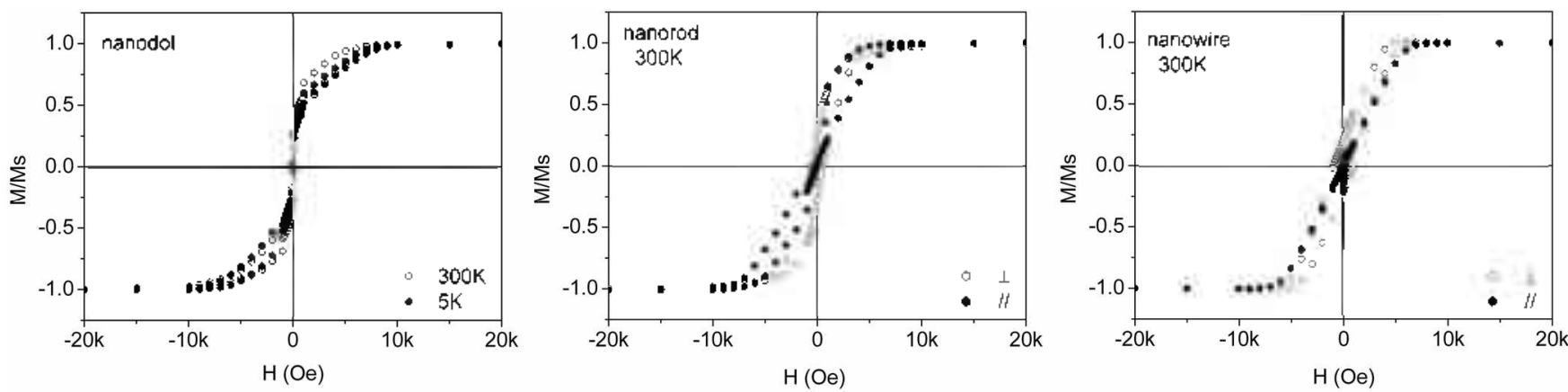

Figure 3. Hy steresis loops for Co (a) nanodots at $5 \mathrm{~K}$ and $300 \mathrm{~K}$. Hysteresis loops for (b) nanorods (c) nanowires measured at $300 \mathrm{~K}$ with the field applied perpendicular ( $O$ ) and parallel $(\bullet)$ to the membrane plane. 


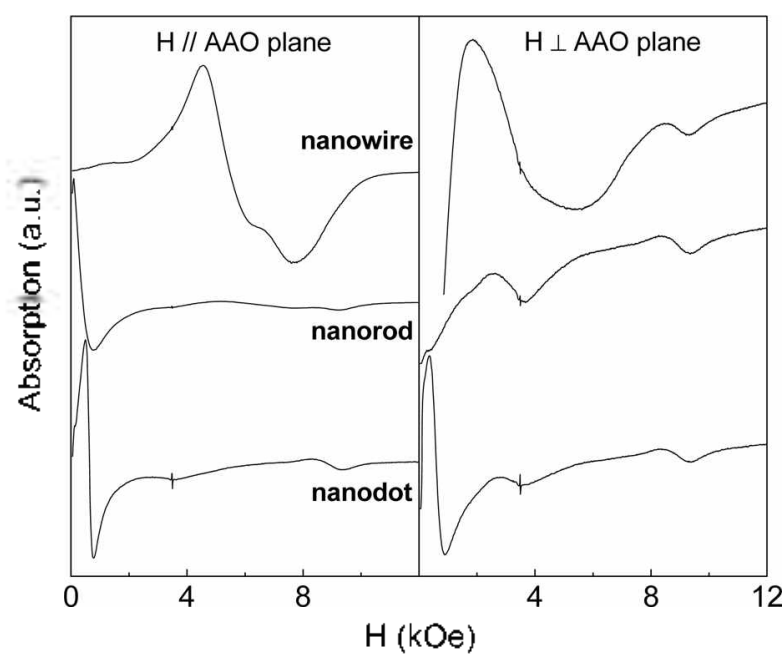

Figure 4. FMR spectra for Co nanodots, nanorods and nanowires with field applied parallel (left panel) and perpendicular (right panel) to the membrane plane.

The differential ferromagnetic resonance spectra measured at room temperature and at the frequency of the nicrowave field of $9.8 \mathrm{GHz}$ with the bias field applied either in the membrane plane or normal to it are displayed in Figure 4. Because of the small amount of magnetic material used for the measurements the microwave signal was weak and small stray resonances, due to sample holder, at about $4 \mathrm{kOe}$ and 9 kOe have been observed. FMR measurements indicate that the resonance fields are strongly affected by the aspect ratio of Co nanostructures. In nanodots, the sharp FMR peak could be clearly observed for the field applied both parallel and perpendicular to the membrane plane. However, in nanorods, FMR peak could be detected only for the field parallel to the membrane plane. The FMR peak becomes broad and shifts to higher field in the Co nanowires.

\section{Conclusion}

We were able to fabricate high quality arrays of cobalt nanostructures with different shapes from dots through rods to wires by controlling time of electrodeposition of $\mathrm{Co}$ in the alumina membranes. The shape of the nanostructures was found to have a significant effect on both static and dynamic properties of the nanostructures.

Acknowledgments. This research was supported by the MIC (Ministry of Information and Communication), Korea, under the 2008 ITRC (Information Technology Research Center) support program supervised by the IITA (Institute of Information Teclunology Assessment) and One of authors (L. Malkinski) acknowledges the support by LEQSF (2005-06)ENH-TR-99 from LABoRSF. Special thanks to Prof. J. Wiley for helpful discussion.

\section{References}

1. Chou. S.: Krauss. P.: Restrom. P. Science 1996. 272. 85.

2. Surn. S.: Murray. C.: Weller. D.: Folks. L.: Moser. A. Science 2000. 287, 1989.

3. Puntes, V. et al. Science 2001. 291, 2115

4. Sauer. G:; Brehm. G.; Schneider. S.; Nielsch, K.: Wehrspohn. R: Choi, J.: Hofmeister, H.: Gosele, U. J. Appl. Phus, 2002, 91. 3243.

5. Zeng. H.: Zheng. M.: Skonnski. R.: Sellmyer. D.: Liu. Y.: Menon. L.: Bandyopadhyay. S. J.Appl. Phos 2000. 87.4718.

6. Vazuez. M.: Pirota. K.: Hernandez-Velez. M.: Prida. V.: Navas. D.; Sanz, R.: Batallan. F.; Velazquerz, J. J. Appl. Phys. 2004, 95. 6642 .

7. O'Connor. C. J. Prog. Inorg. Chem. 1982. 29, 203.

8. Pan1. H.: Liu. B.: Y. J.: Poh. C.: Lim. S.: Ding. J.: Feng. Y.: Huan. C.: Lin. T. J. Phis. Chem. B 2005. 109. 3094.

9. Sellmyer. D. T.: Zheon. M.: Skomski. R. J. Phus:Condens. Manter: 2001. 13, R433.

10. Ursache, A.: Goldbach. J. Y: Russell, 1. P; luorrinen, M. I. $d$. Appl. Plins. 2005, 97. 10J322. 